\title{
Citicolina, ¿deberíamos seguir indicándola en ECV isquémica?
}

\author{
Franklin E Echezuría M, ${ }^{*}$ Rosanna J Quijada M*
}

\section{RESUMEN}

La enfermedad cerebrovascular (ECV) isquémica es una condición médica que implica, según su grado, discapacidad importante para el paciente, además de altos costos para su tratamiento agudo y crónico, así como en el manejo de la discapacidad con alta incidencia y prevalencia a nivel mundial. Desde el punto de vista fisiopatológico, representa una catástrofe en el funcionamiento cerebral que involucra daño estructural y funcional. Se han desarrollado múltiples estrategias para el manejo de esta patología; actualmente, el estándar de oro para su manejo es el rt-PA; sin embargo, por sus características, pocos pacientes tienen acceso al mismo. Razón por la que se han desarrollado estrategias farmacológicas diversas para su manejo: la citicolina se ha usado durante años, no obstante, genera controversias dado que su utilidad, demostrada en estudios experimentales, no se ha reproducido en la práctica clínica; más aún, algunos estudios sugieren que podría empeorar el pronóstico del paciente, lo que justificaría el abandono de su uso en el tratamiento de ECV isquémica (ictus). Por tal motivo ofrecemos esta revisión del tema con el fin de brindar herramientas que permitan aclarar dicha controversia. Palabras clave: Enfermedad cerebrovascular isquémico, ictus, citicolina, neuroprotección.

\section{SUMMARY}

Ischemic cerebrovascular disease (CVD) is a medical condition that, according to its extension, implies significant disability for the patient, as well as high costs for its acute and chronic treatment, as well in the management of disability, with high incidence and worldwide prevalence, from the physiopathological point of view represents a catastrophe in brain functioning that involves structural and functional damage. Multiple strategies have been developed for the management of this pathology, currently the gold standard for its management is the rt-PA, however, due to its characteristics, and few patients have access to it, because it various pharmacological strategies have been developed. Citicoline has been used for years, nevertheless generates controversies since its usefulness, demonstrated in experimental studies, has not been reproduced in clinical practice but some studies suggest that it could worsen the patient's prognosis, which would justify the abandonment of its use in the treatment of ischemic CVD (ictus), for this reason we offer this review of the subject in order to provide tools to clarify this controversy. Key words: Ischemic cerebrovascular disease, ictus, citicoline, neuroprotection.

\section{RESUMO}

A doença cerebrovascular ( $D C b V)$ isquêmica é uma condição clínica que de acordo com sua extensão, implica em uma significativa incapacidade para o paciente, ademais de altos custos para seu tratamento agudo e crônico, bem como no manejo da incapacidade com alta incidência e prevalência a nível mundial. Do ponto de vista fisiopatológico representa uma catástrofe no funcionamento do cérebro que envolve danos estruturais e funcionais. Múltiplas estratégias têm sido desenvolvidas para o tratamento dessa patologia, atualmente o padrão ouro para o seu manejo é o rt-PA, porém, devido às suas características, poucos pacientes têm acesso a ele, razão pela qual várias estratégias farmacológicas foram desenvolvidas, a citicolina vem sendo utilizada há anos, gera controvérsias, uma vez que sua utilidade, demonstrada em estudos experimentais não foi reproduzida na prática clínica, mas alguns estudos sugerem que ela poderia piorar o prognóstico do paciente, o que justificaria o abandono do seu uso no tratamento de DCbV isquêmica (ictus), por esse motivo oferecemos esta revisão, a fim de fornecer ferramentas para esclarecer esta controvérsia. Palavras-chave: DCbV isquêmica, ictus, citicolina, neuroproteção.

\section{INTRODUCCIÓN}

Anualmente, 22 millones de personas sufren ECV isquémica (ictus); a pesar de que las tasas de mortalidad por ictus han disminuido en las últimas dos décadas, ha aumentado el número absoluto de pacientes con

\footnotetext{
* Centro Médico Mazzarri Rey, El Tigre, Estado Anzoátegui, Venezuela.
}

Recepción: 01/12/2017. Aceptación: 07/02/2018.

Este artículo puede ser consultado en versión completa en http://www.medigraphic.com/medicinacritica
ECV isquémicas, muertes relacionadas e indicadores de discapacidad secundaria a esta patología. ${ }^{1,2}$ Además, constituye la segunda causa de discapacidad en países industrializados. ${ }^{3}$ En algunos casos; la discapacidad después de ECV isquémica se considera como peor resultado que la muerte para muchos pacientes y familiares. ${ }^{4}$ En tal contexto, existen múltiples grupos que trabajan en alternativas terapéuticas ante tan devastadora condición patológica.

En la actualidad, se describen dos formas de tratamiento para el ictus: la recanalización del vaso ocluido y la protección del daño al tejido cerebral producida por la isquemia. ${ }^{5}$ La trombólisis con activador tisular del plasminógeno (rt-PA) administrado en las primeras 4-6 horas de inicio de la sintomatología es el tratamiento de elección aprobado por la administración de drogas y alimentos (FDA) en ictus; 6 sin embargo, alrededor de $80-85 \%$ de los pacientes con ictus no cumple con los criterios para el uso de esta terapéutica, ${ }^{7}$ por lo cual las estrategias dirigidas a protección del tejido cerebral en isquemia (neuroprotección) son un importante punto de apoyo en el tratamiento del ictus.

Entendiendo por neuroportección cualquier estrategia que tenga como objetivo antagonizar, interrumpir o enlentecer la cascada de eventos bioquímicos, que, de no ser tratados, resultarían en daño cerebral irreversible, la citicolina, dado su mecanismo de acción, ha sido propuesta como tratamiento «neuroprotector». La citicolina (Somazina ${ }^{\circledR}$ ), nombre genérico de la citidina 5'-difosfocolina (CDP-colina), es un componente esencial para la biosíntesis de fosfolípidos. Es un nucleótido que funciona como intermediario en tres vías metabólicas principales, primero: síntesis de fosfatidilcolina, uno de los principales fosfolípidos de membrana y con un papel importante en la formación de lipoproteínas; segundo: la citicolina provee de colina para la síntesis de acetilcolina, y tercero: actúa como donador de metil durante procesos de oxidación..$^{8,9}$ Sus primeros usos fueron registrados en 1974 por Manaka y cols. como parte del tratamiento en la enfermedad de Parkinson. ${ }^{10}$ Años más tarde, Harrocks y cols. estimaron su posible uso como neuroprotector a partir de la observación de su acción reversible en las fosfotransferasas a nivel cerebral. ${ }^{11}$ Su primera indicación como tratamiento neuroprotector en pacientes con desórdenes neurovegetativos se describió en $1981 .{ }^{12}$

Desde entonces, múltiples estudios se han realizado a fin de validar la utilidad de la citicolina como neuroprotector, con resultados diversos, generando más dudas y controversia sobre sus beneficios en pacientes con 
ictus. Por ello, nos hemos planteado llevar a cabo una revisión del tema, para lo cual realizamos búsquedas en las principales bases de datos de literatura médica (PubMed, Ovid, Cochrane y Scopus), utilizando palabras clave como neuroprotección, citicolina, neuroprotección, entre otras, mediante las cuales tratamos de aclarar los posibles efectos «neuroprotectores» de la citricolina, además de proponer herramientas que permitan aclarar la controversia, para ofrecer un mejor esquema terapéutico y manejo de recursos en pacientes con ictus.

\section{¿Qué es la citicolina?}

Como se describió anteriormente, la citicolina es el nombre de citidina 5'-difosfocolina (Figura 1), la cual es componente exógeno, idéntico al producido naturalmente, fundamental en el metabolismo de fosfolípidos de membrana como fuente de colina en la vía metabólica para la síntesis de acetilcolina y fosfatidilcolina

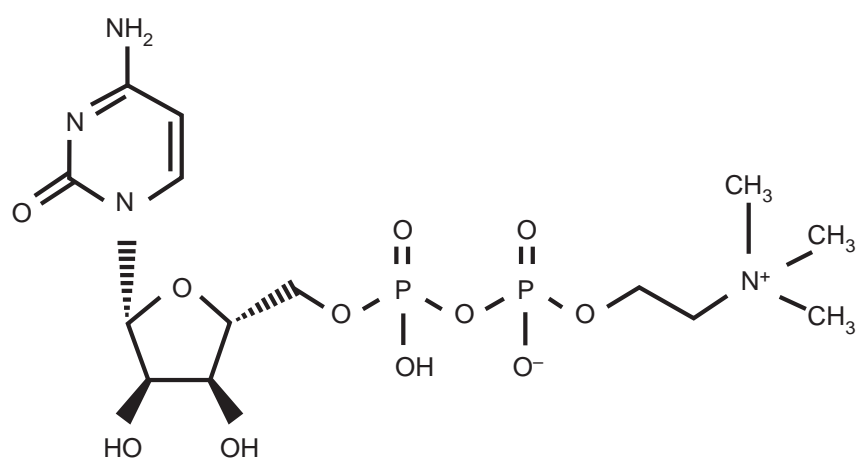

Figura 1: Estructura química de la citicolina, se muestra la citidina a la izquierda y la disfosfocolina (trimetil-etanol-amonio) a la derecha. La citidina es un nucleósido formado por citosina unida a un anillo de ribosa.

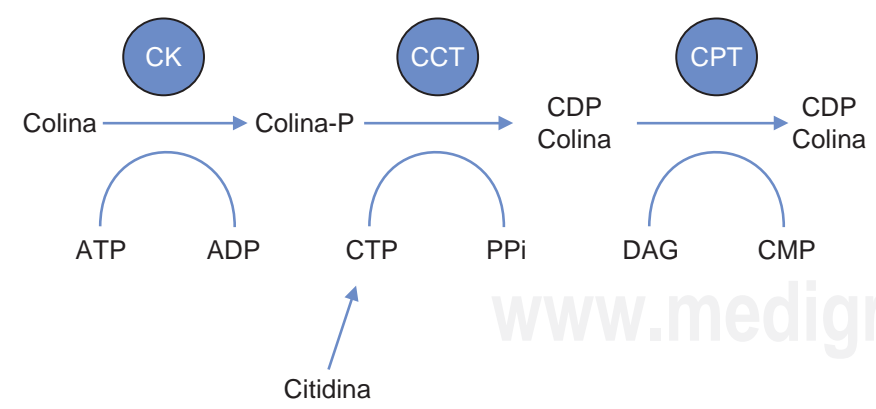

$\mathrm{ADP}=$ Adenosin difosfato, $\mathrm{ATP}=$ Adenosín trifosfato, $\mathrm{CCT}=$ Colinafosfato citidiltransferasa, $\mathrm{CK}=$ Citidin kinasa, CMP = Citidina monfosfato, CPT CDP-colina $=1,2$-diacilglicerolcolinatransferasa, CTP $=$ Citidina trifosfato, $\mathrm{DAG}=1,2$-diacilglicerol, $\mathrm{PC}=$ Fosfatidilcolina, $\mathrm{PP}=$ Pirofosfato.

Adaptado de: Grieb P. Neuroprotective properties of citicoline: facts, doubts and unresolved issues. CNS Drugs. 2014;28(3):185-193.

Figura 2: Vía metabólica de la CDP-colina para la síntesis de fosfatidilcolina. en un proceso de múltiples pasos metabólicos, en el que la citicolina actúa como donador de colina y citidina (Figura 2). ${ }^{13,14}$

En cuanto al metabolismo de la citicolina exógena (Figura 3), existen aspectos sin resolver, al ser administrada oral o parenteralmente, la citicolina se convierte rápidamente en catabolitos colinérgicos. En ratas perfundidas con citicolina marcada, desaparece del hígado en 10 minutos. Estos mismos estudios demuestran que, al ser administrada vía oral, sólo $2 \%$ atraviesa la barrera hematoencefálica, mientras que, con la administración vía endovenosa, alcanza hasta $12 \% .{ }^{15}$ Dado que los compuestos fosforilados se consideran sustancias incapaces de traspasar la membrana celular, se asume que la citicolina (citidina difosfato colina) es desfosforilada en el espacio intravascular en citidina monofosfato y fosfocolina, en el paso siguiente la citidina y la colina son desfosforiladas por las fosfatasas plasmáticas. ${ }^{16}$ Posteriormente, la citidina es transportada al tejido cerebral, donde es resintetizada a citicolina por la CDP fosfocolina-citidiltransferasa para formar parte de la membrana celular.

\section{¿Qué sabemos sobre la utilidad de la citicolina?}

Como se señaló anteriormente, desde la década de los 70 se está hablando de los posibles usos de la citicolina como neuroprotector; estos usos se han probado en múltiples patologías, como traumatismo encéfalo craneano, enfermedad de Parkinson, demencias vasculares, glaucoma y, más recientemente, en enfermedad de Alzheimer $;{ }^{17}$ sin embargo, sus resultados han sido poco

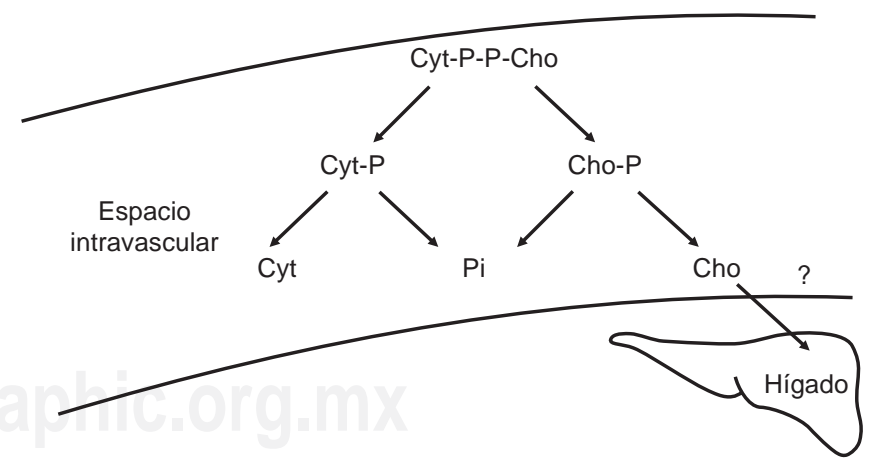

Adaptado de: Grieb P. Neuroprotective properties of citicoline: facts, doubts and unresolved issues. CNS Drugs. 2014;28(3):185-193.

Figura 3: Metabolismo propuesto para la citicolina (Cyt-P-P-Cho) en el espacio intravascular de roedores. En el primer paso, ocurre la hidrólisis de los puentes fosfato; en el segundo paso, la citidina monofosfato (Cyt-P) y la fosfocolina (Cho-P) son desfosforiladas en citidina (Cyt) y colina (Cho), respectivamente. Se cree que buena parte de la colina liberada es captada por el hígado, lo que podría explicar la baja toxicidad colinérgica de la citicolina. 
concluyentes, por lo que su utilidad no se ha extendido en la práctica médica.

En cuanto a su uso en ictus, los datos son bastante controversiales; en estudios experimentales llevados a cabo en ratas tal como el de Gutiérrez, ${ }^{18}$ se utilizó una dosis de $1,000 \mathrm{mg} / \mathrm{kg}$, administrada intraperitonealmente 24 horas postembolización de la arteria carótida derecha y se comparó con la administración de rt-PA a 5 $\mathrm{mg} / \mathrm{kg}$; al comparar estas dos estrategias terapéuticas no se encontraron diferencias estadísticamente significativas en cuanto a la recuperación funcional. Con respecto al tamaño de la lesión visualizada en los cortes histológicos, se evidenció un tamaño menor para el grupo de citicolina $(p<0.05)$. No se evaluó mortalidad en este estudio debido al tipo de trabajo, además, entre sus conclusiones, reportó que la citicolina a altas dosis tiene efecto protector contra la isquemia local, lo anterior basado en la disminución del volumen del tejido afectado; asimismo, señaló que, si bien no era objeto del estudio medir mortalidad, se observó un importante número de muertes relacionadas con complicaciones hemorrágicas.

Le Poncin-Lafitte et al., ${ }^{19}$ en un modelo experimental de isquemia aguda inducida en ratas, valoraron el edema cerebral y la integridad de la barrera hematoencefálica (BHE) con yodoalbúmina marcada y el metabolismo cerebral mediante estudios histoenzimológicos. En este estudio, con la administración de citicolina, se consiguió reducir el edema cerebral vasogénico y restablecer la integridad de la BHE. También comprobaron que con la citicolina el tamaño de los infartos inducidos era menor y que disminuía la actividad de la lactato deshidrogenasa, la succinato deshidrogenasa, la monoaminooxidasa y la fosfatasa ácida y destacaron su papel protector por acción directa en la membrana celular.

Al estudiar los efectos de varios activadores del metabolismo cerebral, Watanabe et al. ${ }^{20}$ observaron que la citicolina aumentaba la incorporación y el metabolismo de la glucosa y disminuía el acúmulo de lactato en el cerebro, además de incrementar levemente el flujo sanguíneo cerebral.

Posterior a la oclusión de las arterias carótidas bilaterales en ratas, Lee et al. ${ }^{21}$ evaluaron el grado de daño de la sustancia blanca y estado cognitivo posterior a la administración de citicolina a dosis de $500 \mathrm{mg} /$ $\mathrm{kg}$ intraperitonealmente por 21 días; los autores concluyeron que la citicolina podía prevenir el daño de la sustancia blanca y ayudar en la mejoría del deterioro cognitivo.

El metaanálisis del grupo de Giralt et al., ${ }^{22}$ en el cual incluyeron 42 trabajos de isquemia inducida en ratas e involucraron un total de 313 animales, destaca una reducción del volumen de isquemia de $28 \%$ (IC 95\%, p $<0.001$ ); asimismo, compara la evolución de animales tratados con dosis de 300 a $500 \mathrm{mg} / \mathrm{kg}$ con animales tratados con dosis de 100 a $300 \mathrm{mg} / \mathrm{kg}$, con evidencia de mayor mejoría en el grupo tratado con dosis altas $27 \%$ versus $18 \%$, respectivamente (IC $95 \% p=0.001$ ).

Tomando en cuenta estos hallazgos, se han llevado a cabo múltiples ensayos clínicos a fin de conocer la utilidad de esta droga.

El grupo de Leon-Jimenez et al. ${ }^{23}$ realizó un estudio caso-control, retrospectivo, multicéntrico en el que se incluyó un total de 87 pacientes con ictus que fueron admitidos a menos de 48 horas del inicio de síntomas, recibieron citicolina $1,000 \mathrm{mg}$ dos veces al día por espacio de dos a cuatro semanas y luego $500 \mathrm{mg}$ dos veces al día por cinco a siete semanas más hasta que completaron nueve semanas de tratamiento y se comparó con un grupo control de similares características epidemiológicas. Entre sus resultados destacan que el grupo que recibió neuroprotección, aunque tuvo menos tiempo de hospitalización y mejoría neurológica, medida según el score de Rankin modificado a los 30 y 90 días, no mostró diferencia estadísticamente significativa.

Por su parte, Clark et al. ${ }^{24}$ realizaron un estudio aleatorizado en el que incluyeron un total de 394 pacientes con ictus moderado según la NIHSS (National Institutes of Health Stroke Scale score), divididos en 267 pacientes que recibieron citicolina a dosis de $500 \mathrm{mg}$ diarios vía oral, iniciando 24 horas desde la aparición del déficit neurológico, y un grupo de 127 que recibió placebo. Al final, concluyeron que a los tres meses de tratamiento continuo no hubo diferencia estadísticamente significativa en el estado neurológico ni volumen de isquemia medido por resonancia magnética. Sin embargo, un análisis post hoc de subgrupos demostró que, en pacientes con ictus de intensidad moderada a elevada definidos por una NIHSS basal superior o igual a ocho, el tratamiento con citicolina mostraba más posibilidades de obtener una recuperación completa a las 12 semanas (21\% placebo, $33 \%$ citicolina; $p=0.05)$, mientras que no se encontraron diferencias en pacientes con ictus de intensidad leve.

En el estudio ECCO $2000^{25}$ se incluyeron 899 pacientes con ictus isquémico agudo de intensidad moderada a elevada (NIHSS $\geq 8$ ), de origen en la arteria cerebral media y con una evolución de menos de 24 horas. Se distribuyó aleatoriamente a los pacientes para recibir $2,000 \mathrm{mg} /$ día de citicolina $(n=453)$ o placebo ( $n=446)$ por vía oral durante seis semanas, con un seguimiento ulterior de seis semanas más. La variable principal del estudio fue el porcentaje de pacientes que al cabo de 12 semanas presentaban una reducción de siete o más puntos en la escala NIHSS. Al final del estudio, $51 \%$ de pacientes del grupo placebo y $52 \%$ del grupo de la citicolina habían conseguido la reducción de siete puntos o más en la escala NIHSS sin que exis- 
tiesen diferencias estadísticamente significativas entre grupos, tampoco hubo diferencia significativa en cuanto a la mortalidad entre los dos grupos.

Otros estudios han observado la reducción en el tamaño de la lesión medida por estudios de neuroimagen, tal es el caso del estudio doble ciego de Warach y cols., ${ }^{26}$ diseñado con el fin medir la evolución del volumen de la lesión isquémica mediante técnicas de difusión ponderada. Se incluyeron 100 pacientes que se distribuyeron aleatoriamente para recibir $500 \mathrm{mg} /$ día de citicolina o placebo por vía oral durante seis semanas. Estos pacientes debían ser incluidos dentro de las primeras 24 horas tras el inicio de los síntomas, tener una puntuación basal en la NIHSS de cinco puntos o más y un volumen lesional en la sustancia gris cerebral de $1,120 \mathrm{~cm}^{3}$ en la RM por difusión ponderada. Las técnicas de neuroimagen se obtuvieron basalmente en las semanas uno y 12. La variable primaria fue la progresión de la lesión isquémica desde la valoración basal hasta la valoración final a las 12 semanas medida por RM. EI análisis primario previsto pudo realizarse en 41 pacientes tratados con citicolina y en 40 pacientes tratados con placebo y no se obtuvieron diferencias significativas.

Dentro del estudio ECCO 2000 se realizó un subestudio para valorar los efectos de la citicolina en el volumen lesional. ${ }^{27}$ En el estudio realizado mediante difusión ponderada se comprobó que; en el grupo placebo $(n=71)$, la lesión se incrementó $30.1 \pm 20.5 \%$, con una mediana de $-8.7 \%$, mientras que el cambio que se produjo en el grupo de la citicolina $(n=63)$ fue de $1.3 \pm$ $14.3 \%$, con una mediana de $-22.9 \%$, sin que se alcanzasen diferencias significativas $(p=0.077)$.

Uno de los estudios más relevantes y recientes en cuanto al uso de la citicolina en ECV isquémico es el estudio ICTUS (International Citicoline Trial on acUte Stroke); ${ }^{28}$ este estudio aleatorizado, caso-control, doble ciego, multicéntrico, realizado entre noviembre de 2006 y octubre de 2011, incluyó 2,298 pacientes de hospitales universitarios de Alemania, Portugal y España con diagnóstico de ECV isquémico e inicio de los síntomas 24 horas antes de su ingreso, con una distribución epidemiológica bastante homogénea, en la que se administró $2,000 \mathrm{mg}$ de citicolina diarios (1,000 mg IV por tres días y luego vía oral) a 1,148 pacientes (49.95\%) y placebo a 1,150 pacientes $(50.04 \%)$ durante seis semanas. El objetivo primario fue evaluar la recuperación neurológica a los 90 días medida por NIHSS, Score de Rankin modificado e índice Barthel. En los resultados de este estudio no se encontraron diferencias entre la recuperación de los pacientes, al punto que el estudio fue detenido por futilidad del tratamiento, ni diferencias significativas en la tasa de efectos adversos relacionados con el tratamiento. La conclusión de los investigadores fue que la citicolina mostró ser segura, pero no efectiva en el tratamiento de ECV isquémico.

\section{¿Entonces, es útil la citicolina en ictus?}

La enfermedad cerebrovascular es una condición con consecuencias devastadoras para el sistema nervioso, que se acompañan de importantes secuelas no sólo para el paciente, sino también para el grupo familiar y altos costos en países donde se llevan programas adecuados de salud pública; por eso, múltiples grupos de investigación abordan el tema para desarrollar estrategias que limiten estos daños.

La citicolina es una de las medidas farmacológicas que buscan ofrecer algún grado de neuroprotección ante tal situación con excelentes resultados en estudios experimentales; sin embargo, estos resultados no han podido ser replicados en ensayos clínicos.

Uno de los elementos que llama la atención al momento de comparar los resultados entre estudios experimentales y ensayos clínicos es la diferencia entre las dosis utilizadas. Como se mencionó anteriormente, las dosis utilizadas en ensayos en ratas son entre 200 y 500 y hasta $1,000 \mathrm{mg} / \mathrm{kg}$, lo que supera por mucho las dosis empleadas en humanos, siendo las dosis entre 1,000 y $2,000 \mathrm{mg} /$ día.

Los ensayos clínicos no demuestran eficacia en la recuperación clínica medida según las escalas validadas internacionalmente (NHISS, score de Rankin modificado o índice Barthel), ni en la disminución del tamaño del área isquémica medida imagenológicamente; no obstante, resaltan el hecho de que la citicolina no muestra efectos adversos importantes en relación con los grupos control.

Por su parte, las guías internacionales para el manejo de ECV isquémico publicadas en marzo de $2013,{ }^{29}$ ratificado en las guías de $2018^{30}$ de la Sociedad Americana de ECV y la Sociedad Americana del Corazón, aparte de «neuroprotección», señalan la futilidad por falta de evidencia que justifique el uso de este tratamiento.

\section{CONCLUSIONES}

Queda claro que la citricolina es un compuesto muy interesante, según sus propiedades farmacológicas con posible utilidad en el marco de ictus; sin embargo, no ha demostrado utilidad en el uso clínico rutinario. A pesar de que las dosis utilizadas en estudios experimentales en roedores (300-500 mg/kg/día) son por mucho superiores a la utilizadas en humanos (1,000-2,000 mg/día) y de que la mayoría de estudios coinciden en que es una droga que carece de efectos colaterales importantes, no hay estudios de dosis equivalentes a los estudios animales (entre 21 y $35 \mathrm{~g} /$ día para un sujeto de $70 \mathrm{~kg}$ ), hecho que podría explicar la falta de resultados de esta droga. Por otro lado, es importante reseñar que los estudios con animales no valoran la recuperación del sujeto, sino los resultados basados en neuroimagen, lo que no 
necesariamente representa recuperación funcional. A la luz de la evidencia actual, la citicolina no debería considerarse como herramienta útil en el tratamiento de una condición tan devastadora. Se requiere más investigación con ajuste de dosis y conocimiento de sus efectos colaterales a dosis que pudiesen ser efectivas en restitución de fosfolípidos de membrana, función celular y mejoría clínica más notable de nuestros pacientes.

\section{BIBLIOGRAFÍA}

1. Feigin VL, Forouzanfar $\mathrm{MH}$, Krishnamurthi $\mathrm{R}$, Mensah GA, Connor M, Bennett DA, et al. Global and regional burden of stroke during 1990-2010: findings from the global burden of disease study 2010. Lancet. 2014;383(9913):245-254.

2. Mukherjee D, Patil G. Epidemiology and the global burden of stroke. World Neurosurg. 2011;76(Suppl. 6):S85-90.

3. Mackay J, Mensah G; World Health Organization. The atlas of heart disease and stroke. Brighton: Myriad Editions Ltd.; 2004.

4. Alvarez-Sabín J, Santamarina E, Maisterra O, Jacas C, Molina C, Quintana M. Long-term treatment with citicoline prevents cognitive decline and predicts a better quality of life after a first ischemic stroke. Int J Mol Sci. 2016;17:390.

5. Labiche LA, Grotta JC. Clinical trials for cytoprotection in stroke. NeuroRx. 2004;1:46-70.

6. Adams H, Adams R, Del Zoppo G, Goldstein LB; Stroke Council of the American Heart Association; American Stroke Association. Stroke Council of the American Heart Association; American Stroke Association. Guidelines for the early management of patients with ischemic stroke: 2005 guidelines update. Stroke. 2005;36(4):916-923

7. Overgaard K. The effects of citicoline on acute ischemic stroke: a review. J Stroke Cerebrovasc Dis. 2014;23(7):1764-1769.

8. Secades JJ, Lorenzo JL. Citicoline: pharmacological and clinical review, 2006 update. Methods Find Exp Clin Pharmacol. 2006;28(Suppl B):1-56.

9. Ulus IH, Wurtman RJ, Mauron C, Blusztajn JK. Choline increases acetylcholine release and protects against the stimulationinduced decrease in phosphatide levels within membranes of rat corpus striatum. Brain Res. 1989;484(1-2):217-227.

10. Manaka S, Sano K, Fuchinoue T, Sekino H. Mechanism of action CDP-choline in parkinsonism. Experientia. 1974;30(2):179-180.

11. Horrocks LA, Dorman RV, Dabrowiecki Z, Goracci G, Porcellat G. CDPcholine and CDP ethanolamine prevent the release of free fatty acids during brain ischemia. Prog Lipid Res. 1981;20:531-534.

12. Horrocks LA, Dorman RV, Dabrowiecki ZM. Therapeutic agents for preventing phospholipid degradation and free fattyacid proliferation. United States Patent. 1981;4:378-386.

13. Adibhatla M, Hatcher F. Citicoline mechanisms and clinical efficacy in cerebral ischemia. J Neurosci Res. 2002;70(2):133139.

14. Parnetti L, Mignini F, Tomassoni D, Traini E, Amenta F. Cholinergic precursors in the treatment of cognitive impairment of vascular origin: ineffective approaches or need for re-evaluation? J Neurol Sci. 2007;257(1-2):264-269.

15. Galletti P, De Rosa M, Cotticelli MG, Morana A, Vaccaro R, Zappia V. Biochemical rationale for the use of CDPcholine in traumatic brain injury: pharmacokinetics of the orally administered drug. J Neurol Sci. 1991;103 Suppl.: S19-25.

16. Galletti P, De Rosa M, Nappi MA, Pontoni G, del Piano L, Salluzzo A, Zappia V. Transport and metabolism of double labelled CDPcholine in mammalian tissues. Biochem Pharmacol. 1985;34(23):4121-4130.
17. Cotroneo AM, Castagna A, Putignano S, Lacava R, Fantò F Monteleone $\mathrm{F}$, et al. Effectiveness and safety of citicoline in mild vascular cognitive impairment: the IDEALE study. Clin Interv Aging. 2013; 8: 131-137.

18. Gutiérrez-Fernández M, Leciñana MA, Rodríguez-Frutos B, Ramos-Cejudo J, Roda JM, Díez-Tejedor E. CDP-choline at high doses is as effective as i.v. thrombolysis. Neurol Res. 2012;34(7):649-956.

19. Le Poncin-Lafitte M, Duterte D, Lageron A, Rapin JR. CDP choline et accident cérébral expérimental d'origine vasculaire. Agressologie. 1986;27(5):413-416.

20. Watanabe S, Kono S, Nakashima Y, Mitsunobu K, Otsuki S. Effects of various cerebral metabolic activators on glucose metabolism of brain. Folia Psychiatr Neurol Jpn. 1975;29:67-76.

21. Lee HJ, Kang JS, Kim YI. Citicoline protects against cognitive impairment in a rat model of chronic cerebral hypoperfusion. $J$ Clin Neurol. 2009;5(1):33-38.

22. Giralt D, García-Bonilla L, et al Selecting the optimal dose of citicoline treatment in Danimal models of focal cerebral ischemia through a meta-analysis. Cerebrovasc Dis. 2010;29(Suppl. 2): 165 .

23. Leon-Jimenez C, Chiquete E, Cantu C, Miramontes-Saldana MJ, Andrade-Ramos MA, Ruiz-Sandoval JL. Citicoline for acute ischemic stroke in mexican hospitals: a retrospective postmarketing analysis. Methods Find Exp Clin Pharmacol. 2010;32(5):325-330.

24. Clark WM, Williams BJ, Selzer KA, Zweifler RM, Sabounjian LA, Gammans RE. A randomized efficacy trial of citicoline in patients with acute ischemic stroke. Stroke. 1999;30(12):2592-2597.

25. Clark WM, Wechsler LR, Sabounjian LA, Schwiderski UE Citicoline Stroke Study Group. A phase III randomized efficacy trial of $2000 \mathrm{mg}$ citicoline in acute ischemic stroke patients. Neurology. 2001;57(9):1595-1602.

26. Warach S, Pettigrew LC, Dashe JF, Pullicino P, Lefkowitz DM, Sabounjian L, et al. Effect of citicoline on ischemic lesions as measured by diffusion weighted magnetic resonance imaging. Ann Neurol. 2000;48(5):713-722.

27. Warach SJ, Sabounjian LA. ECCO 2000 study of citicoline for treatment of acute ischemic stroke: effects on infarct volumes measured by MRI. Stroke. 2000;31:283.

28. Dávalos A, Alvarez-Sabín J, Castillo J, Díez-Tejedor E, Ferro J, Martínez-Vila E, et al. Citicoline in the treatment of acute ischaemic stroke: an international, randomised, multicentre, placebo-controlled study (ICTUS trial). Lancet. 2012;380(9839):349-357

29. Jauch EC, Saver JL, Adams HP Jr, Bruno A, Connors JJ, Demaerschalk BM, et al. Guidelines for the early management of patients with acute ischemic stroke a guideline for healthcare professionals from the American Heart Association/American Stroke Association. Stroke. 2013;44:870-947.

30. Powers WJ, Rabinstein CA, Teri Ackerson VC, Adeoye OM, Bambakidis NC, Becker K, Biller J, Brown M, et al. 2018 Guidelines for the early management of patients with acute ischemic stroke. Stroke. 2018;49:e46-e99.

Conflicto de intereses: Los autores manifestamos no tener ningún conflicto de intereses.

Correspondencia:

Franklin E Echezuría M

Centro Médico Mazzarri Rey,

El Tigre, Estado Anzoátegui, Venezuela. 